\title{
Combined Diagnostic Significance of Preoperative Serum B2-Microglobulin and Routine Blood Test in Patients with High-Grade Glioma and Solitary Brain Metastasis [Erratum]
}

$\mathrm{Li} \mathrm{L}, \mathrm{Bu} \mathrm{X}, \mathrm{Wu} \mathrm{B}$, et al. Cancer Manag Res. the initial submission stage instead of the final revised 2020;12:11735-11742.

The authors have advised that Table 1 on page 11737 and versions. These errors were introduced by the Editorial staff during the publication process. The correct Tables 1

Table 2 on page 11739 are the versions uploaded during and 2 are shown below.

Table I Clinical Characteristic of the Patients with HGG and sBM

\begin{tabular}{|l|l|l|l|}
\hline Parameters & HGG $(\mathbf{n = 1 2 7 )}$ & SBM (n=174) & P \\
\hline Age (years) & $56.00(50.00,65.00)$ & $63.00(53.00,68.00)$ & 0.000 \\
Gender (male/female) & $67 / 60$ & $102 / 72$ & 0.347 \\
$\beta 2-\mathrm{m}(\mu \mathrm{L} / \mathrm{L})$ & $1771.00(1544.00,2063.00)$ & $2067.00(1649.75,2701.00)$ & 0.000 \\
WBC count $\left(\times 10^{9} / \mathrm{L}\right)$ & $6.00(5.10,7.50)$ & $6.10(5.00,7.80)$ & 0.905 \\
Neutrophils $\left(\times 10^{9} / \mathrm{L}\right)$ & $3.90(3.10,5.00)$ & $4.20(3.20,5.70)$ & 0.174 \\
Lymphocytes $\left(\times 10^{9} / \mathrm{L}\right)$ & $1.60(1.30,2.10)$ & $1.40(1.10,1.80)$ & 0.000 \\
Monocytes $\left(\times 10^{9} / \mathrm{L}\right)$ & $0.40(0.30,0.50)$ & $0.40(0.30,0.60)$ & 0.380 \\
Platelets $\left.\times 10^{9} / \mathrm{L}\right)$ & $207.00(166.00,237.00)$ & $204.50(159.00,240.25)$ & 0.670 \\
RDW (\%) & $12.80(12.30,13.20)$ & $13.00(12.40,13.50)$ & 0.052 \\
PDW (\%) & $16.10(15.80,16.40)$ & $16.00(15.70,16.30)$ & 0.305 \\
NLR & $2.38(1.84,3.27)$ & $3.00(2.20,4.39)$ & 0.000 \\
MLR & $0.23(0.19,0.30)$ & $0.29(0.21,0.40)$ & 0.000 \\
\hline
\end{tabular}

Notes: Data were presented as median (25th, 75th percentile).

Abbreviations: HGG, high grade glioma; sBM, solitary brain metastasis; $\beta 2-\mathrm{m}, \beta 2$-microglobulin; WBC, white blood cell; RDW, red cell distribution width; PDW, platelet distribution width; NLR, neutrophil/lymphocyte ratio; MLR, monocyte/lymphocyte ratio.

Table 2 Diagnostic Value of Preoperative Age, $\beta 2-m$, NLR, MLR and Their Combinations in Patients with HGG and sBM

\begin{tabular}{|c|c|c|c|c|c|c|}
\hline Markers & Formula & Cut-off & AUC & $95 \% \mathrm{Cl}$ & Sensitivity (\%) & Specificity (\%) \\
\hline Age (years) & & 56.50 & 0.625 & $0.561-0.689$ & 70.70 & 52.85 \\
\hline$\beta 2-\mathrm{m}(\mu \mathrm{g} / \mathrm{L})$ & & 1932.50 & 0.655 & $0.594-0.717$ & 58.05 & 72.36 \\
\hline NLR & & 2.45 & 0.634 & $0.57 \mid-0.698$ & 71.26 & 52.85 \\
\hline MLR & & 0.28 & 0.622 & $0.559-0.686$ & 56.32 & 71.54 \\
\hline Age $+\beta 2-m$ & $-4.262+0.049 * A g e+0.01 * \beta 2-\mathrm{m}$ & 0.628 & 0.683 & $0.623-0.742$ & 50.57 & 82.11 \\
\hline Age+NLR & $-4.349+0.065 * \mathrm{Age}+0.212 * \mathrm{NLR}$ & 0.541 & 0.683 & $0.622-0.744$ & 71.26 & 43.90 \\
\hline Age+MLR & $-4.291+0.064 * A g e+2.263 * M L R$ & 0.650 & 0.673 & $0.612-0.734$ & 47.13 & 82.11 \\
\hline$\beta 2-m+N L R$ & $-3.3 I I+0.00 I * \beta 2-m+0.225 * N L R$ & 0.621 & 0.720 & $0.663-0.778$ & 55.17 & 86.18 \\
\hline$\beta 2-m+M L R$ & $-2.926+0.00 I * \beta 2-m+2.282 * M L R$ & 0.592 & 0.698 & $0.638-0.757$ & 57.47 & 80.49 \\
\hline$N L R+M L R$ & $-0.923+1.514 * M L R+0.136 * N L R$ & 0.554 & 0.634 & $0.57 \mid-0.697$ & 59.20 & 67.48 \\
\hline Age $+\beta 2-m+N L R$ & $-5.185+0.048 * A g e+0.001 * \beta 2-\mathrm{m}+0.235 * \mathrm{NLR}$ & 0.586 & 0.729 & $0.672-0.785$ & 64.37 & 75.61 \\
\hline Age $+\beta 2-m+M L R$ & $-4.894+0.049 * A g e+0.00 I * \beta 2-\mathrm{m}+2.145 * \mathrm{MLR}$ & 0.569 & 0.716 & $0.658-0.773$ & 65.52 & 72.36 \\
\hline Age $+N L R+M L R$ & $-4.470+0.064 *$ Age $+1.199 * M L R+0.146 * N L R$ & 0.541 & 0.687 & $0.627-0.747$ & 75.29 & 55.28 \\
\hline$\beta 2-m+N L R+M L R$ & $-3.334+0.001 * \beta 2-\mathrm{m}+0.219 * \mathrm{NLR} \quad 0.63 \mathrm{I} * \mathrm{MLR}$ & 0.619 & 0.720 & $0.663-0.777$ & 56.32 & 84.55 \\
\hline Age $+\beta 2-m+N L R$ & $-5.213+0.048 * A g e+0.00 I * \beta 2-\mathrm{m}+0.20 \mathrm{I} * \mathrm{NLR}$ & 0.600 & 0.731 & $0.675-0.788$ & 60.92 & 77.24 \\
\hline$+M L R$ & $+0.594 * M L R$ & & & & & \\
\hline
\end{tabular}

Abbreviations: HGG, high grade glioma; sBM, solitary brain metastasis; $\beta 2-\mathrm{m}, \beta 2$-microglobulin; NLR, neutrophil/lymphocyte ratio; MLR, monocyte/lymphocyte ratio; AUC, area under the curve. 


\section{Publish your work in this journal}

Cancer Management and Research is an international, peer-reviewed open access journal focusing on cancer research and the optimal use of preventative and integrated treatment interventions to achieve improved outcomes, enhanced survival and quality of life for the cancer patient.
The manuscript management system is completely online and includes a very quick and fair peer-review system, which is all easy to use. Visit http://www.dovepress.com/testimonials.php to read real quotes from published authors. 